\title{
A monolithic high-g SOI-MEMS accelerometer for measuring projectile launch and flight accelerations
}

\author{
Bradford S. Davis ${ }^{\mathrm{a}, *}$, Tim Denison ${ }^{\mathrm{b}}$ and Jinbo Kuang ${ }^{\mathrm{b}}$ \\ ${ }^{\mathrm{a}}$ US Army Research Laboratory, Aberdeen Proving Ground, MD 21005, USA \\ ${ }^{\mathrm{b}}$ Analog Devices, Inc., Cambridge, MA 02139, USA
}

Received 14 February 2005

Revised 5 October 2005

\begin{abstract}
Analog Devices (ADI) has designed and fabricated a monolithic high-g acceleration sensor (ADXSTC3-HG) fabricated with the ADI silicon-on-insulator micro-electro-mechanical system (SOI-MEMS) process. The SOI-MEMS sensor structure has a thickness of $10 \mathrm{um}$, allowing for the design of inertial sensors with excellent cross-axis rejection. The high-g accelerometer discussed in this paper was designed to measure in-plane acceleration to $10,000 \mathrm{~g}$ while subjected to $100,000 \mathrm{~g}$ in the orthogonal axes. These requirements were intended to meet Army munition applications. The monolithic sensor was packaged in an 8-pin leadless chip carrier (LCC-8) and was successfully demonstrated by the US Army Research Laboratory (ARL) as part of an inertial measurement unit during an instrumented flight experiment of artillery projectiles launched at 15,000 g.
\end{abstract}

Keywords: Accelerometer, acceleration, balloting, SOI, high-g, MEMS, IMU, telemetry, munition, flight test

\section{Introduction}

The development of the ADI accelerometer was funded through the DARPA MEMS Program, Microsystem Technology Office. The first half of the paper describes the sensor development to include the fabrication process, sensor design, circuit design, and packaging. At the conclusion of the project, ARL received some samples to demonstrate the ADXSTC3-HG's survivability and performance on gun-launched artillery projectiles instrumented with a telemetry-based measurement system. The ADXSTC3-HG's are well suited for ballistic measurement applications because of their small size, low-cost, high-g range, high bandwidth, and good cross-axis rejection capability. The measurement system, theory, and experimental results are discussed in the later half of the paper.

\section{Fabrication of the high-g sensor}

The ADXSTC3-HG sensor was fabricated with the proprietary ADI SOI-MEMS process. The ADI SOI-MEMS process is an extension of the work by Brosnihan [1,2] and can be broken down into four major steps. Referring to Fig. 1, the first process step is to create the SOI-substrate. The substrate is composed of a handle wafer, a 1-um silicon dioxide layer, and a 10-um epitaxial silicon layer. The purpose of the handle wafer is to provide a mechanical foundation for the substrate, the oxide layer serves as a sacrificial layer for releasing the sensor, and the sensor and

\footnotetext{
*Corresponding author. Tel.: +1 410306 0738; Fax: +1 410306 0724; E-mail: bdavis@arl.army.mil.
} 


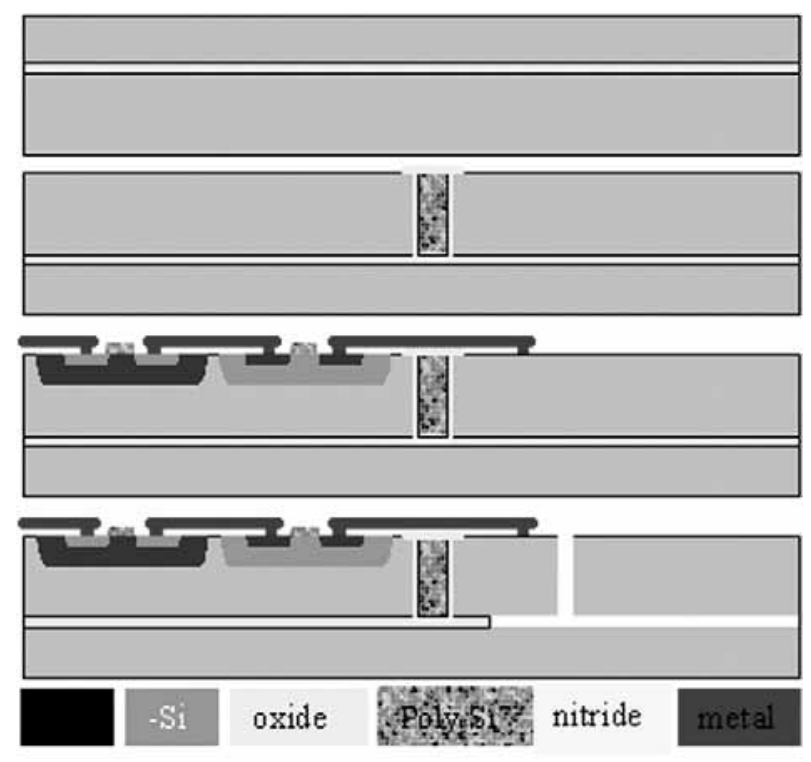

Fig. 1. Simplified SOI-MEMS process flow.

circuitry will be defined on the 10-um epitaxial. The second process step is to use trench isolation to define insulated islands of silicon. The trenches serve to electrically isolate, but mechanically couple, the sensor region and the surrounding epitaxial substrate. Trenches can also be used to electrically isolate regions of the moving sensor to provide for differential sensing architectures. In the third process step, the sensor conditioning circuitry is fabricated using standard sub-micron complementary metal-oxide semiconductor processing. The final process step completes the definition of the sensor by forming the air gaps and releasing the structure by etching away the sacrificial oxide layer. The sensors can then be singulated, packaged, and screened for functionality. The LCC-8 package was chosen for its robustness while undergoing high-g accelerations.

\section{Sensor design}

The design of the sensor focused on achieving robust lateral acceleration measurements $(+/-10 \mathrm{~kg})$ in the presence of large cross-axis disturbances $(+/-100 \mathrm{~kg})$. To achieve robust measurement, the sensor was designed to maximize the aspect ratio of the spring tethers and thereby maximize modal separation. In order to maximize the aspect ratio of the key tethers, the lateral spring of the sensor was split into eight tethering sub-blocks, with four springs per block (see Fig. 2). Electrical connections to the sensor are made through substrate contacts in the isolated pockets defined by the U-shaped trenches. The 5:1 aspect ratio of the springs allowed for a factor of 3 mode separation, which coupled with the distributed placement of the tether sub-blocks provided robust rejection of cross-axis disturbances. The mode separation was limited by stress release mechanisms on the central sensor beam. The cross-axis susceptibility of the sensor was estimated by ANSYS, Inc. design software to be capped at $1.5 \%$. Although the cross-axis rejection could be improved by further increasing the aspect ratio of the tethers, in practice, it is currently limited by the LCC- 8 assembly process to roughly $3 \%$.

The distribution of additional isolation trenches within the sensor design helped to maximize rejection of electrical and mechanical disturbances. Rejection of electrical disturbances, such as the charge injection of the switched capacitor interface, was achieved by designing a fully-differential sensor and interface. As seen in the sensor layout, the moving sensor was bisected by five isolation trenches. These trenches allowed for the differential readout of a single mechanical sensor with minimal excess parasitic capacitance compared to alternative schemes. The rejection of mechanical disturbances required stiffening the fixed fingers attached to the epitaxial layer of the die. In order to increase the stiffness of the fixed fingers, two isolation trenches were used to mechanically couple the fingers and 
Table 1

ADXSTC3-HG specifications

\begin{tabular}{ll}
\hline Full scale acceleration (X axis) & $+/-10 \mathrm{~kg}$ \\
\hline Max cross-axis acceleration (Z axis) & $100 \mathrm{~kg}$ \\
Cross-axis sensitivity & $<3 \%$ \\
Number of sense fingers & 40 \\
Spring constant in X axis, in Z axis & $351 \mathrm{~N} / \mathrm{m}, 3060 \mathrm{~N} / \mathrm{m}$ \\
Resonant frequency in X axis, in Z axis & $84 \mathrm{kHz}, 248 \mathrm{kHz}$ \\
Nominal voltage input, nominal current draw & $5 \mathrm{~V}, 2.5 \mathrm{~mA}$ \\
Frequency Response (3 dB Bandwidth) & $25 \mathrm{kHz}$ \\
\hline
\end{tabular}

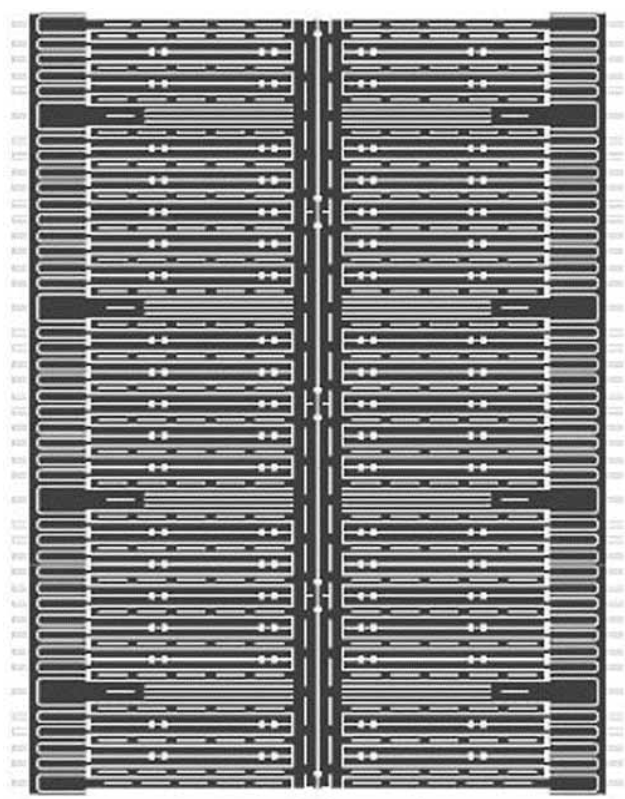

Fig. 2. Layout of the ADXSTC3-HG sensor.

increase the effective finger width by two. The increased thickness increased the resonance frequency of the fixed finger to $248 \mathrm{kHz}$, resulting in a fixed-finger deflection of only $15 \mathrm{~nm}$ in the presence of a $10-\mathrm{kg}$ lateral acceleration. A robust lateral accelerometer with high cross-axis rejection was designed by exploiting the high aspect ratio and trench isolation capabilities of the SOI-MEMS process. The key mechanical and electrical properties of the sensor are summarized in Table 1.

\section{Circuit design}

The ADXSTC3-HG has all signal conditioning integrated monolithically with the sensor (see Fig. 3). The major blocks of interest include a fully-differential sensor interface and low-pass Bessel filter. The interface circuit's function is to convert the sensor's differential capacitance into an analog voltage. Conversion is achieved by using a feedback approach that superimposes a common-mode clock potential, $V_{\mathrm{cm}}$, on top of the differential clock voltages, $V_{d r}$, applied to the sensor's fixed fingers [3]. The feedback loop's integrator adjusts $V_{c m}$ until there is zero net charge transfer from the sense capacitors, $C_{s 1}$ and $C_{s 2}$, into the integration capacitor, $C_{f}$. This charge balancing constraint requires $V_{c m}=V_{d r}\left[\left(C_{s 1}-C_{s 2} / C_{s 1}+C_{s 2}\right)\right]$, which is equivalent to $V_{c m}=V_{d r}\left(\delta / g_{o}\right)$, where $\delta$ is the deflection of the sensor and $g_{o}$ is the nominal gap. The use of the feedback architecture has several advantages including the linear mapping of the output voltage, $V_{c m}$, to sensor displacement and the elimination of non-linear electrostatic forces. The output of the high-bandwidth interface is conditioned by a fully-differential 3-pole switched capacitor Bessel 


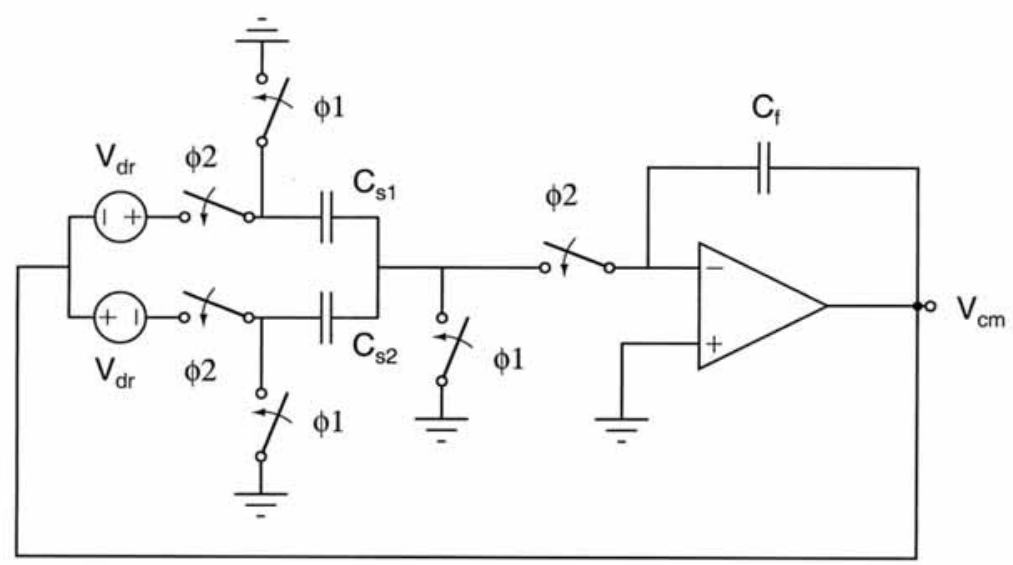

Fig. 3. Interface circuit representation.

filter with a $-3 \mathrm{~dB}$ corner at $25 \mathrm{kHz}$. The architecture of the filter allows for in-package trimming of sensitivity, range, and offset with poly-silicon fuse technology.

\section{Packaging}

A robust packaging scheme was implemented to insure that high-g, high-frequency accelerations would be accurately measured by the accelerometer. The fabricated accelerometers $(2 \times 2$-mm die $)$ were packaged in the LCC-8 and singulated with the ADI punch-tape scheme as shown in Fig. 4. To help improve cross-axis rejection and electrostatic capture, conducting die attach and a pin-to-paddle downbond were used to bias the handle wafer at the nominal sensor voltage.

\section{On-board telemetry instrumentation}

The ADXSTC3-HG's were then used by ARL to measure in-bore acceleration behavior and in-flight projectile spin rate during a flight experiment of $155 \mathrm{~mm}$ M483A1 artillery projectiles at Aberdeen Proving Ground, MD, in December 2003. The accelerometers were mounted with their sense axes in the radial direction on an inertial sensor suite (ISS) board that was packaged within the Diagnostic Fuze (DFuze) (see Fig. 5). The ISS is a double-sided printed circuit board made from commercial off-the-shelf MEMS and other commercially available sensors and their signal conditioning. The DFuze is a patented on-board, telemetry-based instrumentation system developed at ARL that contains g-qualified miniature sensors, microelectronics, data acquisition, a power supply, and telemetry components $[4,5]$. DFuze data verify flight stability and performance, provide on-board diagnostics, validate aerodynamics, and can be used as truth measurements for inertial guidance packages.

\section{Accelerometer measurements}

Spin rate of a projectile in a body-fixed system is defined as the angular velocity of the $J$ - $K$ axes about the $I$ axis. The DFuze uses several different techniques that can estimate the spin rate but none measure it exactly. Two of these techniques are fixed to the sun or earth. For instance, solar sensors are used to measure the projectile's roll rate past the sun and magnetic sensors are used to measure the projectile's roll rate past the Earth's magnetic 


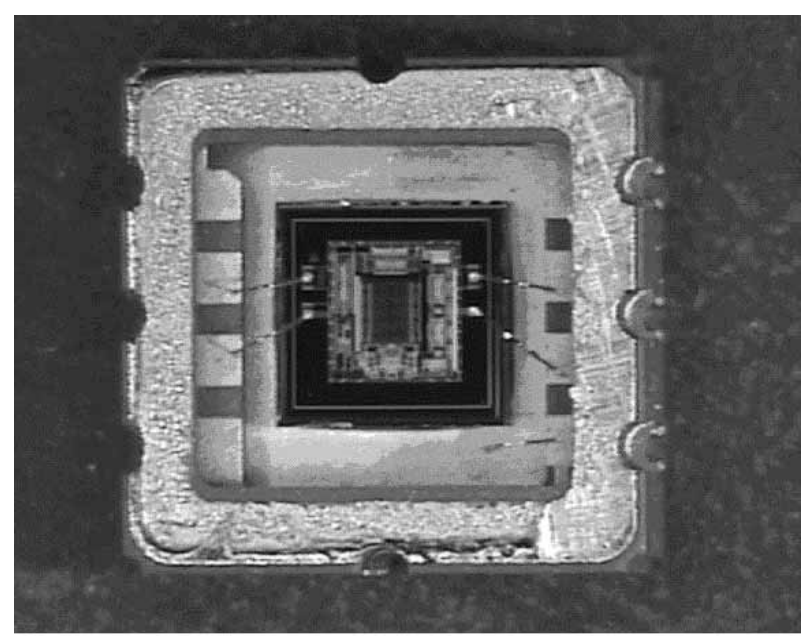

Fig. 4. ADXSTC3-HG sensor die wire bonded to the LCC-8 package.

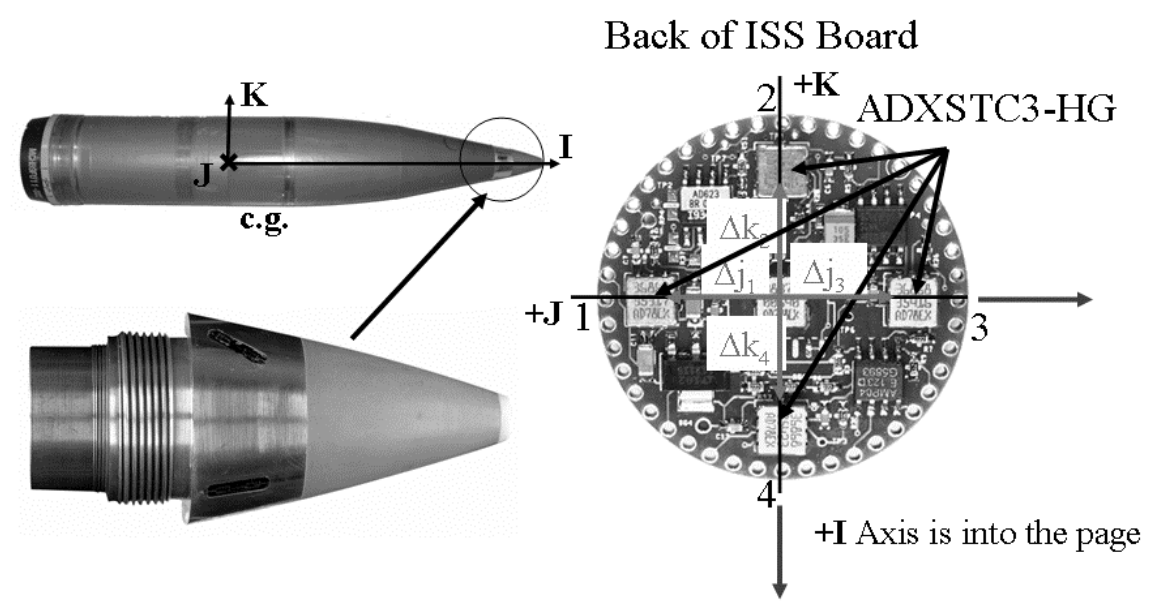

Fig. 5. DFuze and ISS with ADXSTC3-HG accelerometers showing the coordinate system.

field. The accelerometer spin ring is a body-fixed technique that uses a linear combination of the output from four radial accelerometers in a single cross-sectional plane to estimate projectile spin rate, $\bar{p}$. The four accelerometers are positioned in opposed pairs about the projectile spin axis with the sensor axes of each pair co-linear with the diameter on which they are located and co-directional in orientation (see Fig. 5). The ADXSTC3-HG sense axes are aligned along the blue lines, opposite the body-fixed $J$ and $K$ axes. Harkins derived the body-fixed sensor equations and explained their correct interpretation and use [6].

The idealized non-gravitational acceleration components parallel to the $J$ and $K$ axes at a point $(\Delta i, \Delta j, \Delta k$ on a flight body are given in Eq. (1) as follows.

$$
\begin{aligned}
& A_{j}=\dot{v}+r u-p w+\Delta i(p q+\dot{r})+\Delta j\left(-p^{2}-r^{2}\right)+\Delta k(q r-\dot{p})+g \cos \theta \sin \phi \\
& A_{k}=\dot{w}+p v-q u+\Delta i(p r-\dot{q})+\Delta j(q r+\dot{p})+\Delta k\left(-p^{2}-q^{2}\right)+g \cos \theta \cos \phi
\end{aligned}
$$

where

- $A_{j}$ and $A_{k}$ are the sensed acceleration components in the $J$ and $K$ directions,

- $p$ and $\dot{p}$ are the angular velocity and acceleration of the $J-K$ axes about the $I$ axis,

- $q$ and $\dot{q}$ are the angular velocity and acceleration of the $I-K$ axes about the $J$ axis, 


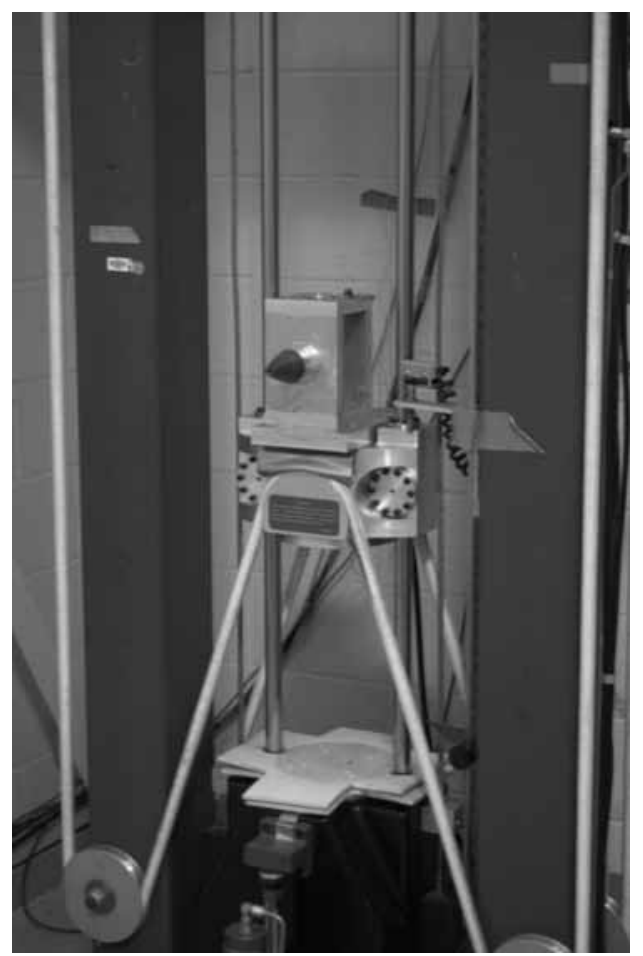

Fig. 6. MTS shock table.

- $r$ and $\dot{r}$ are the angular velocity and acceleration of the $I-J$ axes about the $K$ axis,

- $\Delta i$ is the distance from the center of gravity (c.g.) along the $I$ axis,

- $\Delta j$ and $\Delta k$ are distances from the spin axis along the $J$ and $K$ axes,

- $g$ is the gravity vector, and

- $v, w, u$ and $\dot{v}, \dot{w}, \dot{u}$ are the respective components of translational velocity and acceleration of the pre-launch c.g.

The differences between two such pairs located on the $J$ axis $\left(A_{1}, A_{3}\right)$ and the $K$ axis $\left(A_{2}, A_{4}\right)$ are

$$
\begin{gathered}
A_{3}-A_{1}=\left(\Delta j_{3}-\Delta j_{1}\right)\left(-p^{2}-r^{2}\right) \\
A_{4}-A_{2}=\left(\Delta k_{4}-\Delta k_{2}\right)\left(-p^{2}-q^{2}\right) . \\
\text { If }\left|\Delta j_{1}\right|=\left|\Delta j_{3}\right|=\left|\Delta k_{2}\right|=\left|\Delta k_{4}\right|=R, \text { then } \\
\bar{p}=\sqrt{\frac{A_{3}-A_{1}+A_{4}-A_{2}}{4 R}}=\sqrt{p^{2}+\frac{r^{2}+q^{2}}{2}} .
\end{gathered}
$$

The average of the two opposing accelerometers along the $J$ and $K$ axis removes the spin effects and yields

$$
\begin{aligned}
& A_{j 1,3}=\frac{\left(A_{1}+A_{3}\right)}{2}=\dot{v}+r u-p w+\Delta i(p q+\dot{r})+\Delta k(q r-\dot{p})+g \cos \theta \sin \phi \\
& A_{k 2,4}=\frac{\left(A_{2}+A_{4}\right)}{2}=\dot{w}+p v-q u+\Delta i(p r-\dot{q})+\Delta j(q r+\dot{p})+g \cos \theta \cos \phi .
\end{aligned}
$$

\section{Experimental results}

Shock table testing on an MTS shock machine of sample accelerometers was performed at ARL in November 2003 to verify survivability up to a $30-\mathrm{kg}$ shock (see Fig. 6). The accelerometers tested were compared to a 


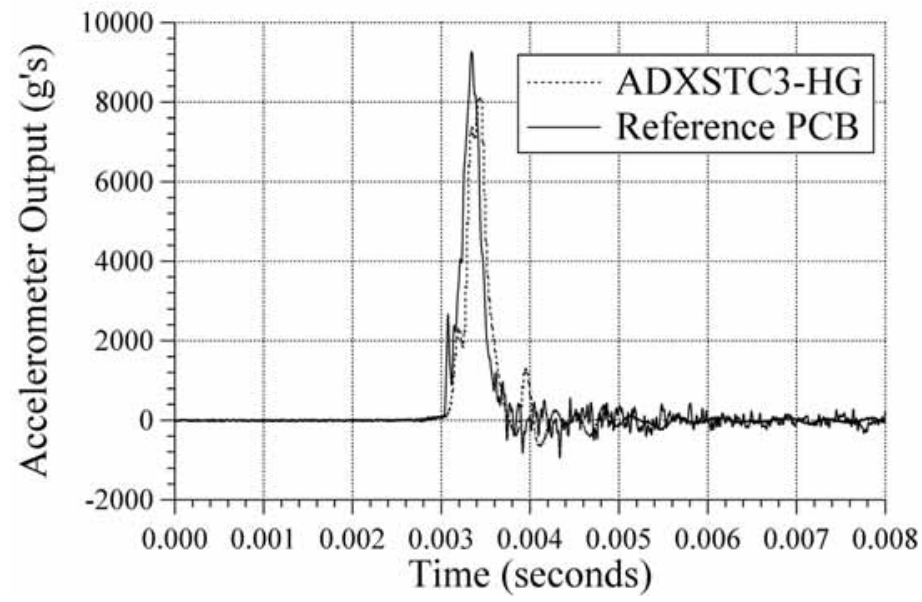

Fig. 7. Shock acceleration vs. time.

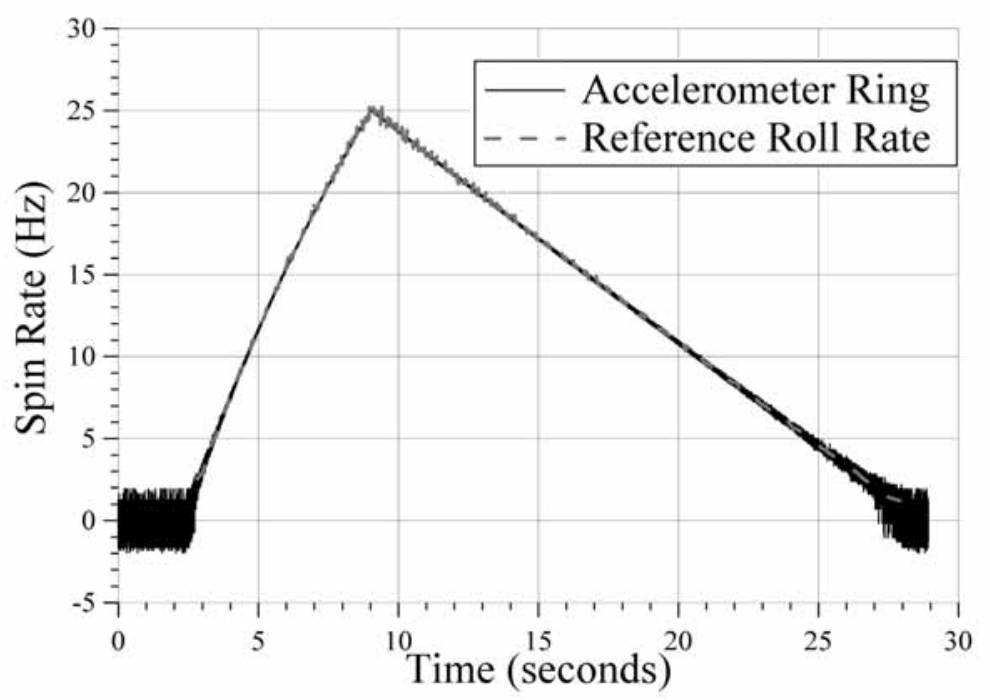

Fig. 8. Spin rate calibration vs. time.

reference accelerometer (PCB model 350B21). Figure 7 shows the acceleration output comparison during a 9$\mathrm{kg}$ shock. Slight differences in amplitude, shape, and timing were noticed. These were most likely a result of mounting differences on the table and the lower bandwidth for the ADI accelerometer as compared to the PCB. All accelerometers passed qualification testing. The accelerometers were then calibrated within the DFuze against a reference spin rate to determine biases and scale factors with a centrifuge prior to flight (see Fig. 7). For the flight experiment, accelerometer rings were tested on four M483A1 projectiles and launched at approximately 2-kg and $15-\mathrm{kg}$ conditions. All accelerometers survived and all data was successfully transmitted and reduced. For brevity, only data from one of the $15-\mathrm{kg}$ experiments will be shown. The output from each individual accelerometer, $A_{1-4}$, was transmitted and scaled using the calibration. The accelerometer outputs were summed on board using digital electronics to provide the accelerometer ring output as a separately transmitted channel. In-bore ballistic phenomena, such as radial acceleration due to balloting and spin-up, was then estimated from the data. Figures 8 and 9 show the radial acceleration and in-bore spin-up measurements the ADX3TCS-HG's were able to provide. In Fig. 10, the projectile's spin rate was measured from the accelerometer ring and compared well to the magnetic roll rate. Errors 


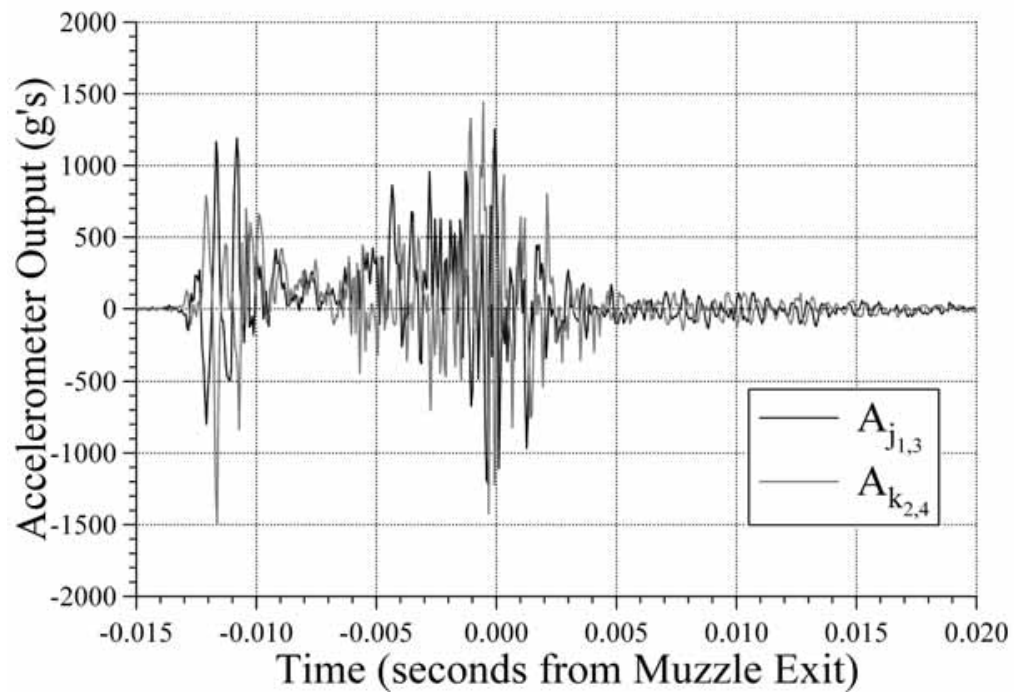

Fig. 9. Projectile balloting vs. time.

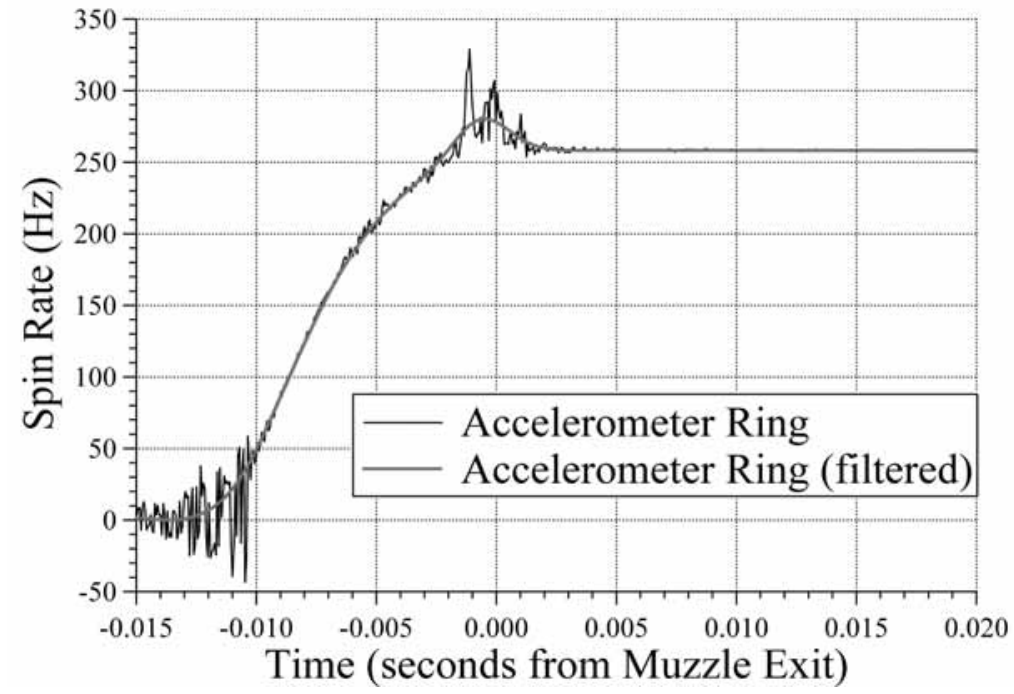

Fig. 10. Projectile spin-up vs. time.

in the measurements can be attributed to accelerometer misalignments, cross-axis sensitivity, and/or the projectile's dynamic behavior.

\section{Conclusions}

Monolithic high-g accelerometers were fabricated by ADI using a SOI-MEMS process under a DARPA-sponsored program. The 10-kg device, packaged in a LCC-8, was designed to operate while subjected to a 100-kg acceleration in the orthogonal axis. ARL successfully demonstrated the ADXSTC3-HG survivability during instrumented experiments on artillery projectiles launched at $15 \mathrm{~kg}$ with a $260-\mathrm{Hz}$ initial spin rate. In-bore ballistic phenomena 


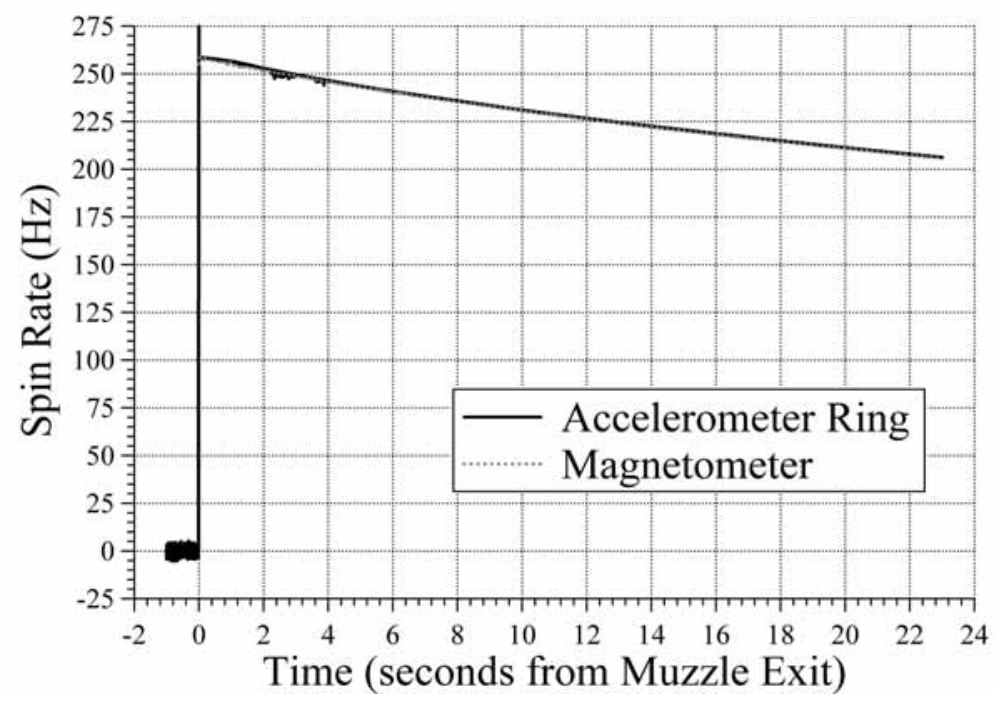

Fig. 11. Projectile spin rate comparison vs. time.

such as spin-up, radial acceleration due to balloting, and spin rate were estimated from the experimental data and compared well to other measurements or theoretical prediction.

\section{Acknowledgments}

The authors would like to thank DARPA-MEMS for funding much of the work. Dr. Thor Juneau is thanked for initiating the early SOI accelerometer work. The authors would like to thank those from ARL: Brendan Patton, David Hepner, and Rex Hall for DFuze assembly; Thomas Harkins for accelerometer ring theoretical modeling; and Gordon Brown and John McLaughlin for shock table testing of the accelerometers. Those from ADI are also thanked: Mike Judy for help in designing the sensor; Dave Collins and the SOI-MEMS development team for their support of the test chip; and Dave Courage for his assistance in packaging the accelerometers.

\section{References}

[1] T. Brosnihan et al., Embedded Interconnect and Electrical Isolation for High-Aspect-Ratio, SOI Inertial Sensors, The 9th International Conference on Solid-State Sensors and Actuators - Transducers '97, June 1997, 637-640.

[2] M. Lemkin, T. Juneau, W. Clark, T. Roessig and T. Brosnihan, A Low-noise Digital Accelerometer Integrated Using SOI-MEMS Technology, Transducers '99, Sendai, Japan, June 7-10, 1999, 1292-1297.

[3] L. Baxter, Capacitive Sensors: Design and Applications, IEEE Press, 1997.

[4] D. Hepner, M. Hollis, P. Muller, T. Harkins, G. Borgen, W. D’Amico, B. Davis and L. Burke, Aeroballistic Diagnostic Fuze, US Patent 6349652, February 2002.

[5] B. Davis, Advancements in G-Hardened Telemetry Instrumentation for Test and Evaluation of Projectiles, Journal of the International Test and Evaluation Association 24(2) (June/July 2003), 51-56.

[6] T. Harkins, Understanding Body-Fixed Sensor Output From Projectile Flight Experiments, US Army Research Laboratory Technical Report 3029, Aberdeen Proving Ground, MD, September 2003. 

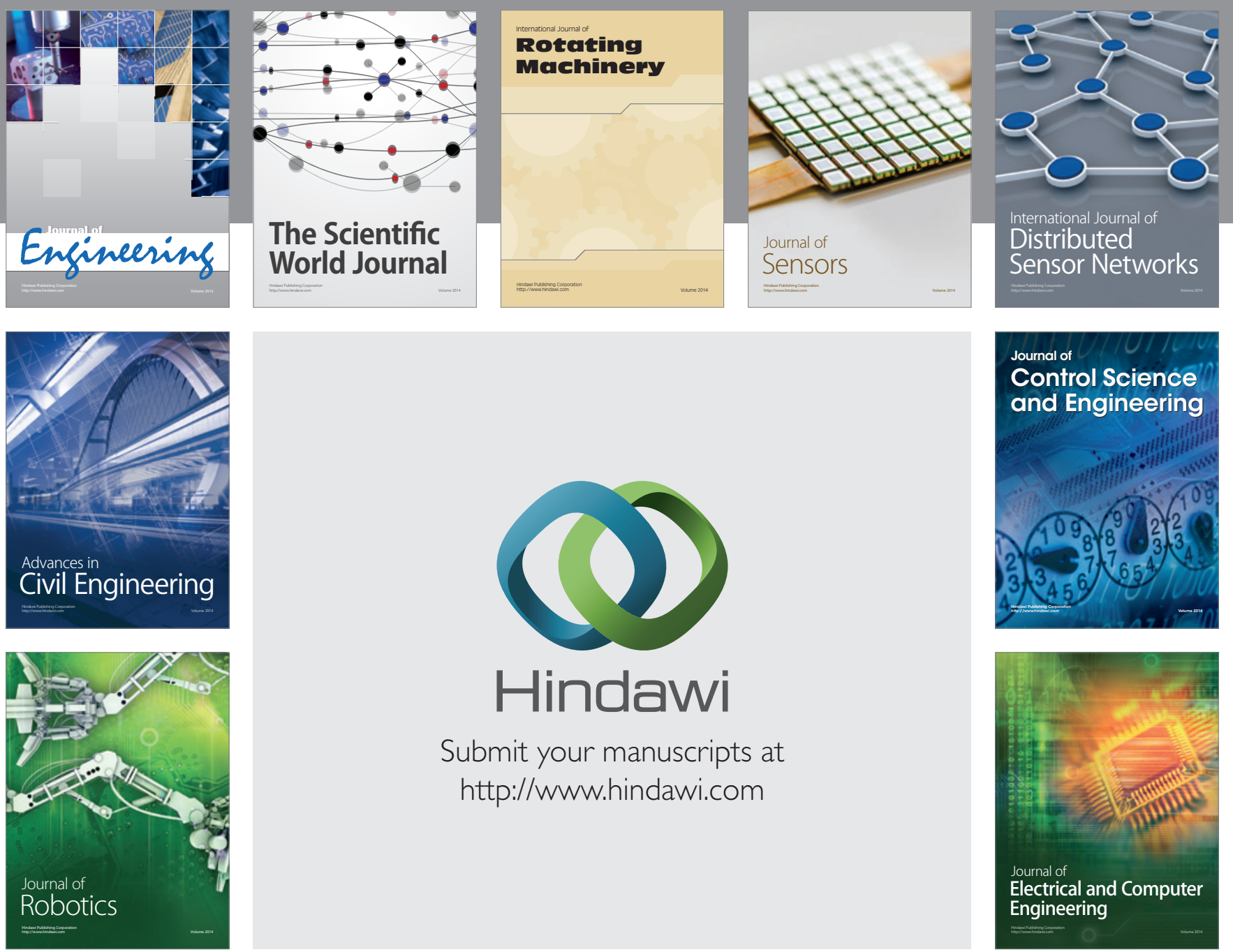

Submit your manuscripts at

http://www.hindawi.com
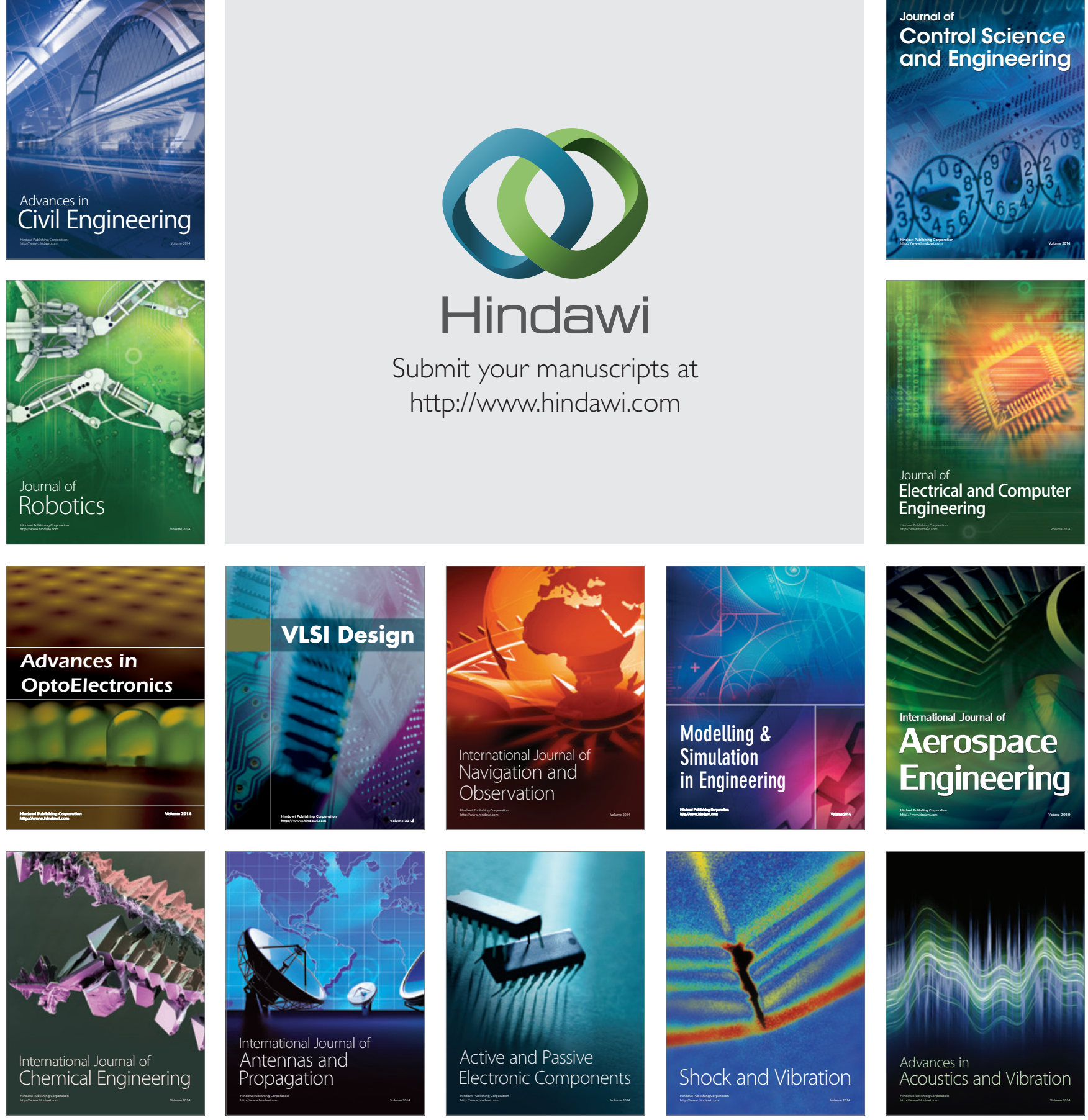